\title{
CLASSICAL ANALOGUE MODELS FOR T AND CPT VIOLATION
}

\author{
ÁGNES ROBERTS \\ Indiana University Physics Department, \\ Bloomington IN 47405, USA \\ E-mail: agrober@indiana.edu
}

\begin{abstract}
Classical-mechanical oscillating systems are analyzed to model CP violating neutral kaon oscillations. Through the identification of the key features of the quantum effective hamiltonian we search for similarities in the characteristic matrix of the classical systems. Some no-go results are found for undamped systems and for systems using two one-dimensional coupled oscillators. A model is presented emulating both $\mathrm{T}$ and spontaneous $\mathrm{CPT}$ violation.
\end{abstract}

\section{Introduction}

Among different quantum oscillating systems, the neutral meson systems are some of the most interesting. Long-standing experimental results indicate that the neutral kaons display a smal violation of the combined $(\mathrm{C})$ charge conjugation and (P) parity symmetries 1 Neutral kaons are now experimentally known to violate $(\mathrm{T})$ time reversal symmetry as well. The formalism for the kaon system, however, could also allow $\mathrm{CP}$ breaking via the violation of the combined CPT symmetry $\mathrm{B}$ resulting from spontaneous CPT breaking ts

One way to analyze these symmetry violations is to model the kaons with a classical analogue system. Simple systems of coupled classical harmonic oscillators can be constructed to parallel the characteristics of the effective hamiltonian describing the kaon oscillations. The kaons and antikaons can be modeled, for instance, using two damped one-dimensional harmonic oscillators weakly coupled through a damped coupling 6 Even though this represents a system which demonstrates the features of the CP eigenstates well, it yields no simple way to model the CP-violating physical states.

Indeed we'll show in the course of this paper that $\mathrm{T}$ violation cannot be modeled via two one-dimensional oscillators in a natural way. The only systems able to model kaon oscillations are those with a single two-dimensional oscillator, an idea also suggested by Rosner In this paper, key features of various classical systems, with corresponding properties of the quantum system, will be analyzed, to create a suitable model for $\mathrm{T}$ and $\mathrm{CPT}$ violation. It will be shown that models with no damping or models with two coupled one-dimensional oscillators cannot fully parallel the characteristic time development of the neutral kaon states. 


\section{Basics}

One goal of this section is to discuss convenient formalisms and basic results. Looking at these results, we can then create a classical analogue model. Following the discussion of the kaon system, the general form of a desired classical model is given.

The general neutral kaon state has a time evolution described by an effective Hamiltonian $\Lambda$ as $i \delta_{t} \Psi=\Lambda \Psi$. The physical kaon states $K_{S}, K_{L}$ are the eigenvectors of $\Lambda$. While $\Lambda$ consists of two Hermitian matrices, $M$ the mass matrix and $\Gamma$ the decay matrix, the combination $\Lambda=M-\frac{1}{2} i \Gamma$ is nonhermitian. This results in a nondiagonalizable $\Lambda$, the eigenstates of which overlap and mix the definite $\mathrm{CP}$ eigenstates, leading to $\mathrm{CP}$ violation.

This feature can be conveniently formulated using the parametrization

$$
\Lambda=\left(\begin{array}{cc}
-i D+E_{3} & E_{1}-i E_{2} \\
E_{1}+i E_{2} & -i D-E_{3}
\end{array}\right),
$$

where $D_{1}, E_{1}, E_{2}, E_{3}$ are complex.

In terms of the above parameters, the condition for $\mathrm{T}$ violation is

$$
\left(\operatorname{Re} E_{2} \operatorname{Im} E_{1}-\operatorname{Re} E_{1} \operatorname{Im} E_{2}\right) \neq 0
$$

and for CPT violation

$$
\operatorname{Re} E_{3} \neq 0 \quad \text { or } \quad \operatorname{Im} E_{3} \neq 0 .
$$

This yields three independent real quantities for the determination of $\mathrm{CP}$ violation in the case of the neutral kaons.

Change in the strong phase of $K^{0}$ and $\overline{K^{0}}$ can mix $E_{1}$ and $E_{2}$ without changing physical behavior. Condition (2), however, is phase invariant 10

Also, the location of the $\mathrm{T}$ and CPT violating parameters can change by choice of a unitarily equivalent new base to the $K^{0}-\overline{K^{0}}$ basis 6 Note, however, that neither of these transformations can mix elements of the mass matrix $M$ and the decay matrix $\Gamma$.

The essential property of the effective hamiltonian is that the CP eigenstates are mixed in the physical states $K_{S}, K_{L}$ because of the particular way $M$ and $\Gamma$ are combined in it.

In modeling the system classically, first we identify $K^{0}-\overline{K^{0}}$ with the two generalized coordinates of a classical harmonic oscillator system.

The individual oscillating frequencies and dampings associated with these two coordinates model the kaon mass energy and decay rate. The equal $K^{0}$ $\overline{K^{0}}$ masses and decay rates thus are represented with oscillators, initially having equal frequency and damping. 
To parallel the idea of spontaneousty broken CPT symmetry in the context of conventional quantum field theory 3 the classical model should also allow an offset of the originally equal frequencies and/or damping of the two generalized coordinates arising dynamically with the full motion of the system. We expect to introduce into our model the analogue for $\mathrm{T}$ violation by coupling the classical oscillators in an appropriate way. This violation can also be emulated spontaneously by the dynamics of the system.

For simplicity, only those models with small oscillations about equilibrium having linear equations of motion are discussed here. Motion of the linear generalized coordinates of such systems can be described by the two component form $Q=\operatorname{Re}\left[A e^{i \omega t}\right]$ with complex $A$. In matrix form, the equation of motion is written as $A X=O . X$ is the classical characteristic matrix. The intention is to create a classical model, the $X$ matrix of which carries features similar to those of $\Lambda$.

\section{Models without damping}

In this section discussing undamped systems, we will establish several no-go results.

The Lagrangian of a conservative classical-mechanical system of small oscillations about equilibrium can be expanded into a quadratic form. The systems considered here have two degrees of freedom, with reabgeneralized coordinate $Q(t)$. A general Langrangian of this kind has the form 9

$$
\mathcal{L}=\frac{1}{2} \dot{Q}^{T} T \dot{Q}+\frac{1}{2} \dot{Q}^{T} G Q-\frac{1}{2} Q^{T} V Q
$$

where $T, G, V$ are $2 \times 2$ matrices. $T, V$ are the symmetric kinetic and potential energy matrices while $\mathrm{G}$, the gyroscopic matrix, is always antisymmetric $\mathrm{\theta}$ The equation of motion of the system is $T \ddot{Q}+G \dot{Q}+V Q=0$. Substituting the solution for $\mathrm{Q}$, the characteristic equation is $X A=0$, giving the characteristic matrix as

$$
X=-\omega^{2} T+i \omega G+V
$$

Inspecting $X$, we see that this type of linear nondissipative system has hermitian characteristic matrices, the eigenvectors of which cannot mix. Hence $X$ cannot model the nonhermitian effective hamiltonian of the kaons. A termby-term comparison between the elements of $X$ and those of $\Lambda$ also shows that all eight parameters of $\Lambda$ cannot be paralleled with a nondissipative system 10 Similarly, $\mathrm{T}$ violation in the neutral kaon system involves dissipative oscillations. 
Comparing $X$ and $\Lambda$, we find that $\operatorname{Re} E_{2}$ is emulated by the gyroscopic term. This property carries over into dissipative systems as well. This motivates us to consider only systems with a gyroscopic matrix. We find, however, that a system with two degrees of freedom with two one-dimensional coupled oscillators cannot produce such terms $₫$ To generate the gyroscopic term, we consider models with time-dependent (rheonomic) constraints. In particular, we look at a model with a uniformly rotating constraint.

\section{Model with viscous damping}

Based on the considerations of the previous sections, we now present a model for both $\mathrm{T}$ and $\mathrm{CPT}$ violation. Take as our model the surface of a bowl spherical on the bottom. A particle oscillates under gravity on the bottom with equal frequencies in two orthogonal directions. With increased heights the cross section of this bowl smoothly transforms into a uniform elliptical shape. When the bowl is rotated with a small amount of friction present, the particle climbs to some equilibrium height determined by gravity and the frequency of rotation. This equilibrium for the elliptical cross section is at either side of the major axis.

After assuming its equilibrium position, the particle rotates with the bowl. The small oscillations it executes vertically and horizontally are both stable. These two oscillations, coupled by the rotation of the bowl, generate a Lagrangian of the form of equation (4), including the gyroscopic matrix.

From the discussion above, we know that dissipation needs to be introduced into the model. To keep the equations of motion linear, we only consider damping describable by the general quadratic expressior

$$
\mathcal{F}=\frac{1}{2} \dot{Q}^{T} R \dot{Q}+\dot{Q}^{T} H Q .
$$

Here, the first term is the Rayleigh dissipation function. The second refers th linear homogeneous damping forces proportional to generalized coordinates 9 The modified equation of motion including damping has the form

$$
T \ddot{Q}+(G+R) \dot{Q}+(V+H) Q=0 .
$$

For the example of the bowl, both such damping terms can be introduced as external viscous damping forces. We can imagine, for instance, a mesh bowl rotating in a static fluid. The fluid exerts resistive force on the particle oscillating inside. This system will produce the desired characteristic matrix 10

$$
X=-T \omega^{2}+i(G+R) \omega+V+H .
$$


Here $T, V, R$ are real symmetric and $G, H$ real antisymmetric matrices The $\omega$ of the damped system is complex. Taking $\omega=\mu+i \kappa$ and defining $\omega^{2}=\Delta^{2}+2 i \mu \kappa$, where $\Delta^{2}=\mu^{2}-\kappa^{2}$, a substitution of $\omega$ into equation (8) gives $X$ to be the sum of the hermitian matrices $-T \Delta^{2}+i \mu G-\kappa R+V$ and the antihermitian matrices $-2 i \mu \kappa T+i \mu R-\kappa G+H$, which is overall nonhermitian. Hence $X$ is nondiagonalizable and has coupled eigenstates. Detailed inspection reveals that there is a difference between diagonal elements of $X$, generating a correspondence to both $\operatorname{Re} E_{3}$ and $\operatorname{Im} E_{3}$ of $\Lambda$. This difference is generated by the rotation of the bowl, emulating spontaneous CPT violation. $X$ is found to

produce an analogue for all eight parameters of $\Lambda 10$

\section{Summary}

This paper studied classical oscillating systems to parallel CP violating neutral kaon oscillations. We found that analogue model without damping and ones with two one-dimensional oscillators cannot be sufficient models. We gave an example showing that properly damped two-dimensional oscillators with timedependent constraint can indeed model spontaneous CPT and $\mathrm{T}$ violation.

\section{References}

1. J.H. Christenson, J.W. Cronin, V.L. Fitch, and R. Turlay, Phys. Rev. Lett. 13138 (1964).

2. For example, R.G. Sachs, The Physics of Time Reversal (University of Chicago Press, Chicago, 1987).

3. T.D. Lee and C.S. Wu, Annu. Rev. Nucl. Sci. 16, 511 (1966).

4. V.A. Kostelecký and R. Potting, Phys. Rev. D 51, 3923 (1995).

5. V.A. Kostelecký and S. Samuel, Phys. Rev. D 39, 683 (1989); 40, 1886 (1989); Phys. Rev. Lett. 63, 224 (1989); 66, 1811 (1991); V.A. Kostelecký and R. Potting, Nucl. Phys. B359, 545 (1991); Phys. Lett. B 381, 89 (1996); Phys. Rev. D 63, 046007 (2001).

6. B. Winstein, in K. Winter, ed., Festi-Val - Festschrift for Val Telegdi (Elsevier, Amsterdam, 1988).

7. J.L. Rosner and S.A. Slezak, hep-ph/9912506.

8. D. Colladay and V.A. Kostelecký, Phys. Rev. D 55, 6760 (1997); Phys. Rev. D 58, 116002 (1998).

9. L. Meirovitch, Principles and Techniques of Vibrations (Prentice-Hall, New Jersey, 1997).

10. Á. Roberts and V.A. Kostelecký, Phys. Rev. D 63, 096002 (2001). 